\title{
Optimal Design of Complex Passive-Damping Systems for Vibration Control of Large Structures: An Energy-to-Peak Approach
}

\author{
Francisco Palacios-Quiñonero, ${ }^{1}$ Josep Rubió-Massegú, ${ }^{1}$ \\ Josep M. Rossell, ${ }^{1}$ and Hamid Reza Karimi ${ }^{2}$ \\ ${ }^{1}$ Department of Applied Mathematics III, Universitat Politècnica de Catalunya (UPC), Avenue Bases de Manresa 61-73, Manresa, \\ 08242 Barcelona, Spain \\ ${ }^{2}$ Department of Engineering, Faculty of Engineering and Science, University of Agder (UiA), 4898 Grimstad, Norway
}

Correspondence should be addressed to Francisco Palacios-Quiñonero; francisco.palacios@upc.edu

Received 25 April 2014; Accepted 4 June 2014; Published 29 June 2014

Academic Editor: Shen Yin

Copyright ( 2014 Francisco Palacios-Quiñonero et al. This is an open access article distributed under the Creative Commons Attribution License, which permits unrestricted use, distribution, and reproduction in any medium, provided the original work is properly cited.

\begin{abstract}
We present a new design strategy that makes it possible to synthesize decentralized output-feedback controllers by solving two successive optimization problems with linear matrix inequality (LMI) constraints. In the initial LMI optimization problem, two auxiliary elements are computed: a standard state-feedback controller, which can be taken as a reference in the performance assessment, and a matrix that facilitates a proper definition of the main LMI optimization problem. Next, by solving the second optimization problem, the output-feedback controller is obtained. The proposed strategy extends recent results in static outputfeedback control and can be applied to design complex passive-damping systems for vibrational control of large structures. More precisely, by taking advantages of the existing link between fully decentralized velocity-feedback controllers and passive linear dampers, advanced active feedback control strategies can be used to design complex passive-damping systems, which combine the simplicity and robustness of passive control systems with the efficiency of active feedback control. To demonstrate the effectiveness of the proposed approach, a passive-damping system for the seismic protection of a five-story building is designed with excellent results.
\end{abstract}

\section{Introduction}

The latest trends in vibration control of large structures consider distributed actuation systems, which mitigate the vibrational response of the overall structure by means of the coordinated actuation of a large number of medium-size semiactive or passive devices [1-3]. In this context, decentralized and semidecentralized control strategies are especially relevant, and fully decentralized velocity-feedback controllers constitute a case of particular interest [4-9]. In addition to the typical advantages of decentralization, fully decentralized velocity-feedback controllers have the singular feature of admitting a passive implementation by means of linear dampers. By taking advantages of this property, advanced active feedback control strategies can be used to design complex passive-damping systems for vibration control of large structures. The passive-damping systems so obtained combine the simplicity and robustness of passive control systems with the effectiveness of active feedback control systems [10-12]. However, from a practical point of view, this approach leads to serious difficulties, mainly associated with the high computational cost of designing decentralized static output-feedback controllers [13-16].

Recently, an effective two-step design methodology to synthesize output-feedback controllers has been proposed in [17]. In the initial step, the goal is to obtain a satisfactory statefeedback controller by solving an optimization problem with linear matrix inequality (LMI) constraints $\mathscr{P}_{s}$. Next, a second LMI optimization problem $\mathscr{P}_{o}$ is derived by introducing in 
$\mathscr{P}_{s}$ a suitable transformation of the LMI variables, and the desired output-feedback controller is computed by solving $\mathscr{P}_{0}$.

The transformations of the LMI variables used to obtain the output-feedback LMI optimization problem $\mathscr{P}_{o}$ from the state-feedback LMI optimization problem $\mathscr{P}_{s}$ contain an arbitrary matrix $L$, which plays a critical role in both the feasibility and the effectiveness of the method $[17,18]$. A simplified version of this design methodology has been successfully applied in designing decentralized velocity-feedback controllers for seismic protection of single buildings [19, 20] and two-building systems [21]. These preliminary works were conducted using a null $L$-matrix and, in all the cases, the corresponding LMI optimization problems $\mathscr{P}_{0}$ were initially reported to be infeasible by the Matlab LMI solver [22]. Although these feasibility issues could be conveniently overcome by using a slightly perturbed state matrix, it became apparent that a more satisfactory solution to the problem was certainly desirable.

After a detailed study of the LMI variables' transformations, an advanced choice of the $L$-matrix has been proposed in [17], which makes it possible to compute centralized velocity-feedback $H_{\infty}$ controllers for structural vibration control with no feasibility issues. This choice, however, fails to produce positive results in the relevant case of fully decentralized velocity-feedback controllers.

Over the last years, the energy-to-peak approach has been attracting an increasing research interest. Some recent applications of this design strategy to structural vibration control and other complex control problems can be found in [21, 23$28]$. In the present paper, we provide a new choice of the $L$ matrix that allows synthesizing fully decentralized velocityfeedback energy-to-peak controllers for structural vibration control of large structures without feasibility issues and with an excellent level of performance. For brevity and clarity, a particular five-story building has been chosen to introduce the main ideas. A more general description of the design procedure can be found in [17-19].

The paper is organized as follows. In Section 2, a mathematical model for the five-story building is provided. Section 3 is devoted to complete the initial step of the design procedure. More specifically, an ideal state-feedback energyto-peak controller is designed, and numerical simulations are conducted to demonstrate its effectiveness. Next, in Section 4, two different velocity-feedback controllers are synthesized. Firstly, a centralized velocity-feedback controller is computed using the L-matrix choice proposed in [17]. Secondly, a fully decentralized velocity-feedback controller is obtained by means of a novel choice of the $L$-matrix. This decentralized controller can be implemented by a set of linear passive dampers, which can operate without sensors, with no communication system and null power consumption. To assess the effectiveness of the proposed passive control system, numerical simulations of the building vibrational response are conducted and compared with the results attained by the ideal active state-feedback controller. Finally, in Section 5, some conclusions and future research directions are briefly presented.

\section{Five-Story Building Model}

Let us consider a five-story building whose lateral motion can be described by the following differential equation:

$$
M \ddot{q}(t)+C_{d} \dot{q}(t)+K_{s} q(t)=T_{u} u(t)+T_{w} w(t),
$$

where

$$
q(t)=\left[q_{1}(t), q_{2}(t), q_{3}(t), q_{4}(t), q_{5}(t)\right]^{T}
$$

is the vector of displacements relative to the ground, $w(t)$ denotes the seismic ground acceleration, and

$$
u(t)=\left[u_{1}(t), u_{2}(t), u_{3}(t), u_{4}(t), u_{5}(t)\right]^{T}
$$

represents the vector of control actions. $M, C_{d}$, and $K_{s}$ are the mass, damping, and stiffness matrices, respectively, $T_{u}$ is the control location matrix, and $T_{w}$ is the excitation location matrix. The following particular values of the matrices $M, C_{d}$, $K_{s}, T_{u}$, and $T_{w}$ are used in the present paper:

$$
\begin{aligned}
& M=10^{3} \times\left[\begin{array}{ccccc}
215.2 & 0 & 0 & 0 & 0 \\
0 & 209.2 & 0 & 0 & 0 \\
0 & 0 & 207.0 & 0 & 0 \\
0 & 0 & 0 & 204.8 & 0 \\
0 & 0 & 0 & 0 & 266.1
\end{array}\right], \\
& C_{d}=10^{3} \times\left[\begin{array}{ccccc}
650.4 & -231.1 & 0 & 0 & 0 \\
-231.1 & 548.9 & -202.5 & 0 & 0 \\
0 & -202.5 & 498.6 & -182.0 & 0 \\
0 & 0 & -182.0 & 466.8 & -171.8 \\
0 & 0 & 0 & -171.8 & 318.5
\end{array}\right] \text {, } \\
& K_{s}=10^{6} \times\left[\begin{array}{ccccc}
260 & -113 & 0 & 0 & 0 \\
-113 & 212 & -99 & 0 & 0 \\
0 & -99 & 188 & -89 & 0 \\
0 & 0 & -89 & 173 & -84 \\
0 & 0 & 0 & -84 & 84
\end{array}\right] \text {, } \\
& T_{u}=\left[\begin{array}{ccccc}
1 & -1 & 0 & 0 & 0 \\
0 & 1 & -1 & 0 & 0 \\
0 & 0 & 1 & -1 & 0 \\
0 & 0 & 0 & 1 & -1 \\
0 & 0 & 0 & 0 & 1
\end{array}\right], \quad T_{w}=-M\left[\begin{array}{l}
1 \\
1 \\
1 \\
1 \\
1
\end{array}\right],
\end{aligned}
$$

where masses are in $\mathrm{kg}$, damping coefficients in $\mathrm{Ns} / \mathrm{m}$, and stiffness coefficients in $\mathrm{N} / \mathrm{m}$. The mass and stiffness values used in the matrices $M$ and $K_{s}$ are similar to those presented in [29], and the damping matrix $C_{d}$ has been computed as a Rayleigh damping matrix with a 5\% damping ratio on the first and fifth modes [30]. We assume that an actuation device $a_{i}$ has been implemented between the consecutive stories $s_{i-1}$ and $s_{i}, i=1, \ldots, 5$. The actuation device $a_{i}$ exerts a control action $u_{i}(t)$, which produces a pair of structural opposite forces as indicated in Figure 1. By considering the state vector,

$$
x_{I}(t)=\left[\begin{array}{l}
q(t) \\
\dot{q}(t)
\end{array}\right],
$$


we can derive a first-order state-space model

$$
\dot{x}_{I}(t)=A_{I} x_{I}(t)+B_{I} u(t)+E_{I} w(t)
$$

with the following system matrices:

$$
\begin{gathered}
A_{I}=\left[\begin{array}{cc}
{[0]_{5 \times 5}} & I_{5} \\
-M^{-1} K_{s} & -M^{-1} C_{d}
\end{array}\right], \\
B_{I}=\left[\begin{array}{c}
{[0]_{5 \times 5}} \\
M^{-1} T_{u}
\end{array}\right], \quad E_{I}=\left[\begin{array}{c}
{[0]_{5 \times 1}} \\
-[1]_{5 \times 1}
\end{array}\right],
\end{gathered}
$$

where $[0]_{n \times m}$ represents a zero-matrix of the indicated dimensions, $I_{n}$ is the identity matrix of order $n$, and $[1]_{n \times 1}$ denotes a vector of dimension $n$ with all its entries equal to 1 . Next, we consider the vector of interstory drifts,

$$
r(t)=\left[q_{1}, q_{2}-q_{1}, \ldots, q_{5}-q_{4}\right]^{T}
$$

and the vector of interstory velocities,

$$
v(t)=\left[\dot{q}_{1}, \dot{q}_{2}-\dot{q}_{1}, \ldots, \dot{q}_{5}-\dot{q}_{4}\right]^{T},
$$

and define the new state vector,

$$
x(t)=\left[\begin{array}{l}
r(t) \\
v(t)
\end{array}\right]
$$

which can be expressed as

$$
x(t)=\mathscr{C} x_{I}(t)
$$

with the change of basis matrix

$$
\mathscr{C}=\left[\begin{array}{cccccccccc}
1 & 0 & 0 & 0 & 0 & 0 & 0 & 0 & 0 & 0 \\
-1 & 1 & 0 & 0 & 0 & 0 & 0 & 0 & 0 & 0 \\
0 & -1 & 1 & 0 & 0 & 0 & 0 & 0 & 0 & 0 \\
0 & 0 & -1 & 1 & 0 & 0 & 0 & 0 & 0 & 0 \\
0 & 0 & 0 & -1 & 1 & 0 & 0 & 0 & 0 & 0 \\
0 & 0 & 0 & 0 & 0 & 1 & 0 & 0 & 0 & 0 \\
0 & 0 & 0 & 0 & 0 & -1 & 1 & 0 & 0 & 0 \\
0 & 0 & 0 & 0 & 0 & 0 & -1 & 1 & 0 & 0 \\
0 & 0 & 0 & 0 & 0 & 0 & 0 & -1 & 1 & 0 \\
0 & 0 & 0 & 0 & 0 & 0 & 0 & 0 & -1 & 1
\end{array}\right] .
$$

The new state-space model can be written as

$$
\dot{x}(t)=A x(t)+B u(t)+E w(t),
$$

with

$$
A=\mathscr{C} A_{I} \mathscr{C}^{-1}, \quad B=\mathscr{C} B_{I}, \quad E=\mathscr{C} E_{I} .
$$

For the particular building matrices given in (4), we obtain the system matrices presented in (15):

$$
\begin{aligned}
& A=10^{3} \times\left[\begin{array}{cccccccccc}
0 & 0 & 0 & 0 & 0 & 0.0010 & 0 & 0 & 0 & 0 \\
0 & 0 & 0 & 0 & 0 & 0 & 0.0010 & 0 & 0 & 0 \\
0 & 0 & 0 & 0 & 0 & 0 & 0 & 0.0010 & 0 & 0 \\
0 & 0 & 0 & 0 & 0 & 0 & 0 & 0 & 0.0010 & 0 \\
0 & 0 & 0 & 0 & 0 & 0 & 0 & 0 & 0 & 0.0010 \\
-0.6831 & 0.5251 & 0 & 0 & 0 & -0.0019 & 0.0011 & 0 & 0 & 0 \\
0.6831 & -1.0652 & 0.4732 & 0 & 0 & 0.0014 & -0.0027 & 0.0010 & 0 & 0 \\
0 & 0.5402 & -0.9515 & 0.4300 & 0 & 0 & 0.0011 & -0.0025 & 0.0009 & 0 \\
0 & 0 & 0.4783 & -0.8645 & 0.4102 & 0 & 0 & 0.0010 & -0.0023 & 0.0008 \\
0 & 0 & 0 & 0.4346 & -0.7258 & 0 & 0 & 0 & 0.0009 & -0.0020
\end{array}\right] \text {, } \\
& B=10^{-5} \times\left[\begin{array}{ccccc}
0 & 0 & 0 & 0 & 0 \\
0 & 0 & 0 & 0 & 0 \\
0 & 0 & 0 & 0 & 0 \\
0 & 0 & 0 & 0 & 0 \\
0 & 0 & 0 & 0 & 0 \\
0.4647 & -0.4647 & 0 & 0 & 0 \\
-0.4647 & 0.9427 & -0.4780 & 0 & 0 \\
0 & -0.4780 & 0.9611 & -0.4831 & 0 \\
0 & 0 & -0.4831 & 0.9714 & -0.4883 \\
0 & 0 & 0 & -0.4883 & 0.8641
\end{array}\right], \quad E=\left[\begin{array}{c}
0 \\
0 \\
0 \\
0 \\
0 \\
-1 \\
0 \\
0 \\
0 \\
0
\end{array}\right] \\
& \widetilde{G}_{s}=10^{7} \times\left[\begin{array}{cccccccccc}
3.6830 & -0.3330 & -1.2296 & -1.6206 & -1.1538 & -0.3751 & -0.2093 & -0.1011 & -0.0347 & -0.0059 \\
2.7397 & 0.3097 & -0.9642 & -1.5512 & -1.1682 & -0.2123 & -0.2741 & -0.1705 & -0.1042 & -0.0526 \\
-1.0352 & 0.5680 & -0.0594 & 0.3626 & 0.1681 & -0.0801 & -0.1139 & -0.1958 & -0.1157 & -0.0663 \\
-3.5761 & -0.2533 & 1.4597 & 1.4264 & 1.4844 & -0.0074 & -0.0206 & -0.0603 & -0.1439 & -0.0616 \\
-3.4161 & -0.5983 & 1.3527 & 2.1085 & 0.9566 & 0.0100 & 0.0139 & -0.0006 & -0.0321 & -0.1092
\end{array}\right] .
\end{aligned}
$$




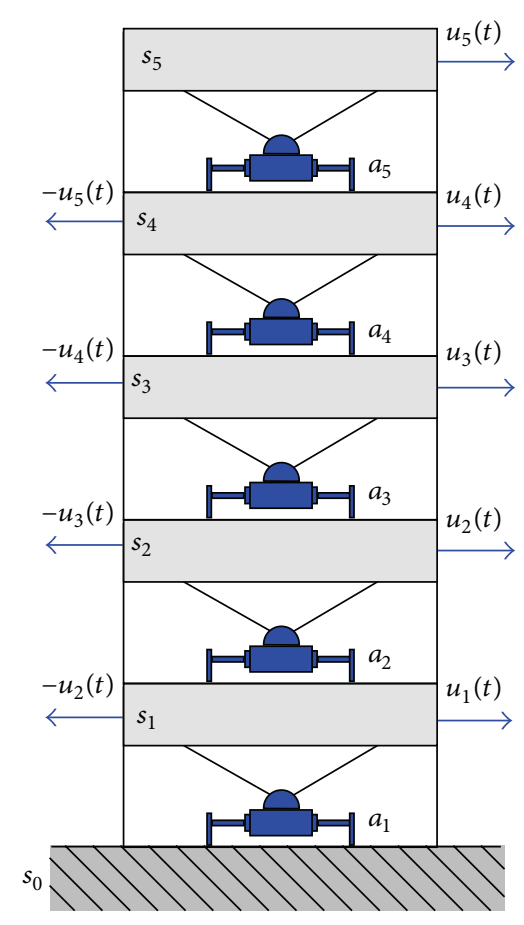

FIGURE 1: Five-story building actuation scheme.

\section{State-Feedback Controller}

The initial step of the design procedure proposed in [17] consists in determining a satisfactory state-feedback controller. This ideal controller has full access to the state information and must satisfy the performance requirements of the problem under consideration.

For the five-story building presented in Section 2, let us assume that the goals in the controller design are minimizing the interstory drifts seismic response and the control efforts. To this end, we introduce the vector of controlled outputs:

$$
z(t)=C_{z} x(t)+D_{z} u(t)
$$

where

$$
C_{z}=\left[\begin{array}{cc}
I_{5} & {[0]_{5 \times 5}} \\
{[0]_{5 \times 5}} & {[0]_{5 \times 5}}
\end{array}\right], \quad D_{z}=\alpha\left[\begin{array}{c}
{[0]_{5 \times 5}} \\
I_{5}
\end{array}\right],
$$

and $\alpha>0$ is a suitable coefficient that trades off the conflicting design objectives. The aim of this section is to compute a state-feedback energy-to-peak controller:

$$
u(t)=\widetilde{G}_{s} x(t)
$$

for the state-space system given in (13), using the controlled output $z(t)$ to define the performance criteria. The energyto-peak control approach considers the largest gain from the disturbance energy to the controlled-output peak:

$$
\gamma_{G}=\sup _{0<\|w\|_{2}<\infty} \frac{\left\|z_{G}\right\|_{\infty}}{\|w\|_{2}},
$$

where $w(t)$ is the input disturbance,

$$
z_{G}(t)=\left(C_{z}+D_{z} G\right) x(t)
$$

is the closed-loop controlled-output corresponding to the state-feedback controller $u(t)=G x(t)$, and

$$
\begin{aligned}
& \|w\|_{2}=\left(\int_{0}^{\infty} w^{T}(t) w(t) d t\right)^{1 / 2}, \\
& \left\|z_{G}\right\|_{\infty}=\sup _{0 \leq t<\infty}\left(z_{G}^{T}(t) z_{G}(t)\right)^{1 / 2} .
\end{aligned}
$$

Broadly speaking, the controller design consists in obtaining a gain matrix $\widetilde{G}_{s}$ that produces an asymptotically stable closed-loop system

$$
\dot{x}(t)=\left(A+B \widetilde{G}_{s}\right) x(t)
$$

and, simultaneously, attains an optimally small $\gamma$-value $\gamma_{\widetilde{G}_{s}}$. These objectives can be achieved by solving the following LMI optimization problem [24]:

$$
\begin{gathered}
\mathscr{P}_{s}:\left\{\begin{array}{l}
\text { minimize } \eta \\
\text { subject to } X>0, \eta>0, \\
\text { and the LMIs in }(25),
\end{array}\right. \\
A X+X A^{T}+B Y+Y^{T} B^{T}+E E^{T}<0, \\
{\left[\begin{array}{cc}
X & * \\
C_{z} X+D_{z} Y & \eta I
\end{array}\right]>0,}
\end{gathered}
$$

where $*$ denotes the transpose of the symmetric entry. If an optimal value $\widetilde{\eta}_{s}$ is attained in $\mathscr{P}_{s}$ for the pair of matrices $\left(\widetilde{X}_{s}, \widetilde{Y}_{s}\right)$, then $\widetilde{G}_{s}$ can be written in the following form:

$$
\widetilde{G}_{s}=\widetilde{Y}_{s} \widetilde{X}_{s}^{-1}
$$

and the optimal $\gamma$-value can be computed as follows:

$$
\gamma_{\widetilde{G}_{s}}=\widetilde{\eta}_{s}^{1 / 2}
$$

By solving the optimization problem $\mathscr{P}_{s}$ with the system matrices $A, B$, and $E$ displayed in (15), the matrices $C_{z}$ and $D_{z}$ defined in (18), and the value

$$
\alpha=10^{-7.55}
$$

we obtain the state-feedback control gain matrix $\widetilde{G}_{s}$ presented in (16) and the optimal $\gamma$-value:

$$
\gamma_{\widetilde{G}_{s}}=0.0395 \text {. }
$$

To provide a better insight into the behavior of the statefeedback controller defined by the gain matrix $\widetilde{G}_{s}$, we have conducted numerical simulations of the five-story building vibrational response, using the full scale North-South $1940 \mathrm{El}$ Centro seismic record as ground acceleration input (see Figure 2). The maximum absolute interstory drifts are displayed in Figure 3, where the blue line with circles corresponds to the state-feedback controller, and the black line with rectangles presents the vibrational response of the uncontrolled building. The maximum absolute control efforts corresponding to the state-feedback controller are displayed 


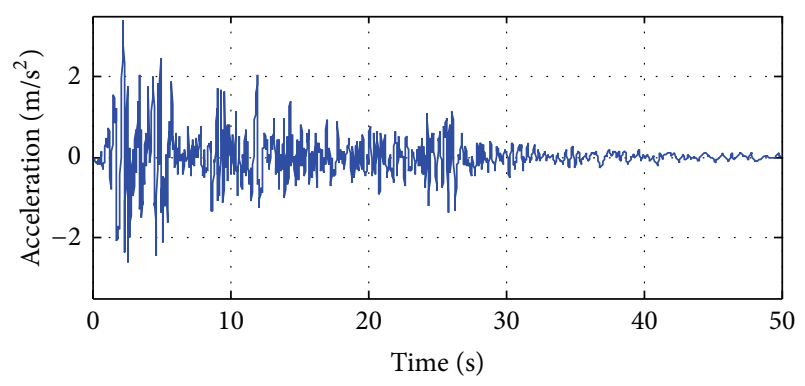

FIgURE 2: Full scale North-South El Centro 1940 seismic record.

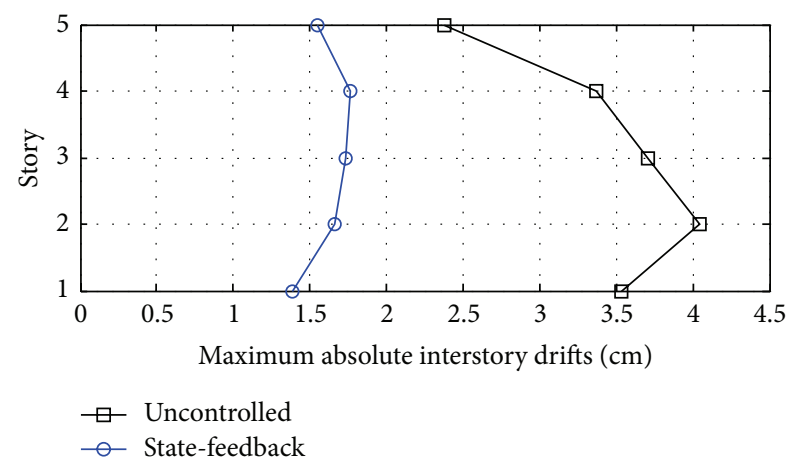

FIGURE 3: Maximum absolute interstory drifts corresponding to the uncontrolled building (black line with rectangles) and the controlled building with the ideal state-feedback energy-to-peak controller defined by the gain matrix $\widetilde{G}_{s}$ (blue line with circles). The full scale North-South El Centro 1940 seismic record has been used as ground acceleration disturbance.

in Figure 4. A quick look at the graphics clearly shows that the proposed state-feedback energy-to-peak controller attains a good level of reduction in the interstory drifts peak-values with moderate levels of control effort. In what follows, we will assume that $\widetilde{G}_{s}$ defines a suitable state-feedback controller for the five- story building introduced in Section 2.

Remark 1. Looking at the graphic in Figure 4, it can be observed that the control actions corresponding to the proposed state-feedback controller present peak-values in the range $0.6-1.0 \mathrm{MN}$. Control forces of this magnitude, or even larger, are commonly used in modern control systems for vibration control of large structures $[1,5]$. For example, control forces of $1 \mathrm{MN}$ can be produced by the semiactive hydraulic dampers implemented in the Kajima Shizuoka building [29], and $2 \mathrm{MN}$ control forces can be obtained with the passive hydraulic damper with semiactive characteristics presented in [31].

\section{Velocity-Feedback Controllers}

In this section, the two-step design procedure proposed in [17] is first applied to synthesize a centralized energy-topeak velocity-feedback controller for the five-story building defined in Section 2. Next, a new choice of the $L$-matrix is presented, which makes it possible to compute a fully

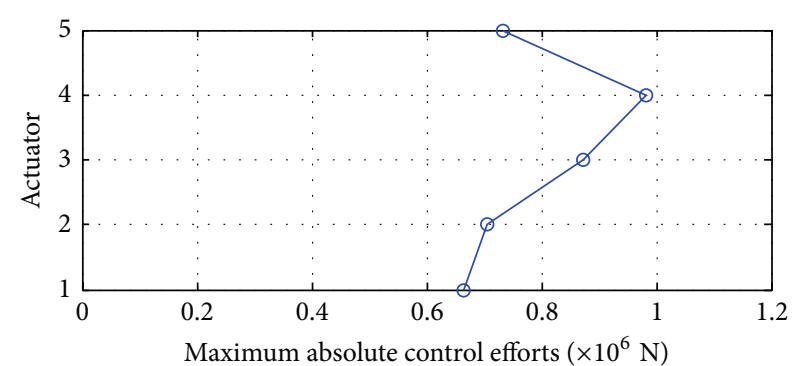

FIGURE 4: Maximum absolute control efforts corresponding to the controlled building with the ideal state-feedback energy-to-peak controller defined by the gain matrix $\widetilde{G}_{s}$. The full scale North-South El Centro 1940 seismic record has been used as ground acceleration disturbance.

decentralized energy-to-peak velocity-feedback controller with no feasibility issues.

4.1. Centralized Velocity-Feedback Controller. In this section, we assume that the information available for feedback purposes is the vector of interstory velocities $v(t)$ defined in (9), which can be written in the form

$$
v(t)=C_{v} x(t)
$$

by taking the observed-output matrix

$$
C_{v}=\left[[0]_{5 \times 5} I_{5}\right] .
$$

In this case, we consider the velocity-feedback controller:

$$
u(t)=\widetilde{K} v(t),
$$

and the energy-to-peak design objective consists in obtaining a gain matrix $\widetilde{K}$ that produces an asymptotically stable closedloop system

$$
\dot{x}(t)=\left(A+B \widetilde{G}_{k}\right) x(t)
$$

and, simultaneously, attains an optimally small $\gamma$-value $\gamma_{\widetilde{G}_{k}}$, where

$$
\widetilde{G}_{k}=\widetilde{K} C_{v}
$$

is the state gain matrix associated with the velocity-feedback controller. According to the ideas presented in [18], the gain matrix $\widetilde{K}$ can be computed by considering the state-feedback LMI optimization problem $\mathscr{P}_{s}$ given in (24) and the following transformations of the LMI variables $X$ and $Y$ :

$$
X=Q X_{Q} Q^{T}+R X_{R} R^{T}, \quad Y=Y_{R} R^{T},
$$

which introduce, as new variables, a square matrix $Y_{R} \in \mathbb{R}^{5 \times 5}$ and two symmetric matrices $X_{\mathrm{Q}} \in \mathbb{R}^{5 \times 5}$ and $X_{R} \in \mathbb{R}^{5 \times 5}$. Two constant matrices, $Q \in \mathbb{R}^{10 \times 5}$ and $R \in \mathbb{R}^{10 \times 5}$, are used to define the LMI variable transformations. $Q$ is a matrix whose columns contain a basis of $\operatorname{Ker}\left(C_{v}\right)$, and the matrix $R$ has the following form:

$$
R=C_{v}^{\dagger}+Q L
$$


where

$$
C_{v}^{\dagger}=C_{v}^{T}\left(C_{v} C_{v}^{T}\right)^{-1}
$$

is the Moore-Penrose pseudoinverse of $C_{v}$, and $L \in \mathbb{R}^{5 \times 5}$ denotes an arbitrary and constant matrix.

By substituting the transformations (35) in (25), we obtain the LMIs:

$$
\begin{aligned}
& A Q X_{Q} Q^{T}+Q X_{Q} Q^{T} A^{T}+A R X_{R} R^{T} \\
& +R X_{R} R^{T} A^{T}+B Y_{R} R^{T}+R Y_{R}^{T} B^{T}+E E^{T}<0, \\
& {\left[\begin{array}{cc}
Q X_{Q} Q^{T}+R X_{R} R^{T} & * \\
C_{z} Q X_{Q} Q^{T}+C_{z} R X_{R} R^{T}+D_{z} Y_{R} R^{T} & \eta I
\end{array}\right]>0 .}
\end{aligned}
$$

A centralized velocity-feedback energy-to-peak controller can now be designed by solving the following LMI optimization problem:

$$
\mathscr{P}_{o}:\left\{\begin{array}{l}
\text { minimize } \eta \\
\text { subject to } X_{\mathrm{Q}}>0, X_{R}>0, \eta>0, \\
\text { and the LMIs in }(38) .
\end{array}\right.
$$

If an optimal value $\tilde{\eta}_{o}$ is attained in $\mathscr{P}_{o}$ for the triplet $\left(\widetilde{X}_{Q}, \widetilde{X}_{R}, \widetilde{Y}_{R}\right)$, then the velocity gain matrix $\widetilde{K}$ can be written in the form

$$
\widetilde{K}=\widetilde{Y}_{R}\left(\widetilde{X}_{R}\right)^{-1},
$$

and the corresponding $\gamma$-value satisfies

$$
\gamma_{\widetilde{G}_{k}} \leq \widetilde{\eta}_{o}^{1 / 2}
$$

By solving the optimization problem $\mathscr{P}_{o}$ with the same matrices $A, B, E, C_{z}$, and $D_{z}$ used in the optimization problem $\mathscr{P}_{s}$ and the following matrices $Q$ and $R$ :

$$
\begin{aligned}
& Q=\left[\begin{array}{lllll}
1 & 0 & 0 & 0 & 0 \\
0 & 1 & 0 & 0 & 0 \\
0 & 0 & 1 & 0 & 0 \\
0 & 0 & 0 & 1 & 0 \\
0 & 0 & 0 & 0 & 1 \\
0 & 0 & 0 & 0 & 0 \\
0 & 0 & 0 & 0 & 0 \\
0 & 0 & 0 & 0 & 0 \\
0 & 0 & 0 & 0 & 0 \\
0 & 0 & 0 & 0 & 0
\end{array}\right] \\
& R=\left[\begin{array}{ccccc}
0.0007 & 0.0086 & 0.0112 & 0.0120 & 0.0089 \\
-0.0260 & -0.0088 & 0.0151 & 0.0235 & 0.0181 \\
-0.0201 & -0.0117 & -0.0006 & 0.0134 & 0.0129 \\
-0.0097 & -0.0078 & -0.0057 & -0.0061 & 0.0004 \\
-0.0014 & -0.0030 & -0.0067 & -0.0084 & -0.0109 \\
1 & 0 & 0 & 0 & 0 \\
0 & 1 & 0 & 0 & 0 \\
0 & 0 & 1 & 0 & 0 \\
0 & 0 & 0 & 1 & 0 \\
0 & 0 & 0 & 0 & 1
\end{array}\right] \text {, }
\end{aligned}
$$

we obtain the velocity gain matrix

$$
\begin{aligned}
\widetilde{K}=10^{6} & \times\left[\begin{array}{cccccc}
-3.0212 & -0.9868 & -0.4766 & -0.4127 & -0.3271 \\
-0.7667 & -2.7879 & -1.6298 & -0.7395 & -0.2145 \\
-0.6874 & -1.3196 & -2.3003 & -1.2415 & -0.5403 \\
-0.7940 & -0.3717 & -1.0121 & -2.1209 & -1.2644 \\
-0.7082 & 0.0873 & -0.2535 & -1.1524 & -1.9441
\end{array}\right]
\end{aligned}
$$

with an associated $\gamma$-value that satisfies

$$
\gamma_{\widetilde{G}_{k}} \leq 0.0397 \text {. }
$$

The matrix $R$ in (43) has been computed using the $L$ matrix:

$$
L=Q^{\dagger} \widetilde{X}_{s} C_{v}^{T}\left(C_{v} \widetilde{X}_{s} C_{v}^{T}\right)^{-1}
$$

where

$$
Q^{\dagger}=\left(Q^{T} Q\right)^{-1} Q^{T}
$$

is the Moore-Penrose pseudoinverse of $Q$ and $\widetilde{X}_{s}$ is the optimal $X$-matrix of the state-feedback optimization problem $\mathscr{P}_{s}$. With this particular choice of the $L$-matrix, it has been possible to solve the LMI optimization problem $\mathscr{P}_{o}$ with no feasibility issues and, moreover, we have obtained a practically optimal velocity-feedback controller. This approach has also been used in [17] to design a centralized velocity-feedback $H_{\infty}$ controller with positive results.

4.2. Fully Decentralized Velocity-Feedback Controller. Now, we are interested in obtaining a fully decentralized velocityfeedback energy-to-peak controller:

$$
u(t)=\widetilde{K}_{d} v(t)
$$

In this case, the gain matrix $\widetilde{K}_{d}$ has a diagonal structure:

$$
\widetilde{K}_{d}=\left[\begin{array}{ccccc}
k_{11} & 0 & 0 & 0 & 0 \\
0 & k_{22} & 0 & 0 & 0 \\
0 & 0 & k_{33} & 0 & 0 \\
0 & 0 & 0 & k_{44} & 0 \\
0 & 0 & 0 & 0 & k_{55}
\end{array}\right],
$$

and, in principle, it could be computed by solving the LMI optimization problem $\mathscr{P}_{o}$, given in (39), with the additional zero-nonzero structure constraints:

$$
\begin{aligned}
& X_{R}=\left[\begin{array}{lllll}
\mathbf{\square} & 0 & 0 & 0 & 0 \\
0 & \mathbf{\square} & 0 & 0 & 0 \\
0 & 0 & \mathbf{\square} & 0 & 0 \\
0 & 0 & 0 & \mathbf{\square} & 0 \\
0 & 0 & 0 & 0 & \mathbf{\square}
\end{array}\right], \\
& Y_{R}=\left[\begin{array}{lllll}
\mathbf{\square} & 0 & 0 & 0 & 0 \\
0 & \mathbf{\square} & 0 & 0 & 0 \\
0 & 0 & \mathbf{\square} & 0 & 0 \\
0 & 0 & 0 & \mathbf{\square} & 0 \\
0 & 0 & 0 & 0 & \mathbf{\square}
\end{array}\right],
\end{aligned}
$$


where the black squares represent the allowed positions for nonzero elements. Unfortunately, this LMI optimization problem with structure constraints is reported to be unfeasible by the Matlab LMI solver, and the same situation happens for the $H_{\infty}$ approach.

It must be observed, however, that the $L$-matrix in (36) is an arbitrary matrix and, consequently, other choices can be made to cope with the encountered feasibility issues. After exploring some slight variations of the $L$-matrix given in (46), a proper solution to the present problem has been obtained by taking an $L$-matrix with the following form:

$$
L_{d}=Q^{\dagger} \widetilde{X}_{s} C_{v}^{T}\left(C_{v} \widetilde{X}_{s}^{(d)} C_{v}^{T}\right)^{-1}
$$

where $\widetilde{X}_{s}^{(d)}$ is a diagonal matrix that contains the diagonal elements of $\widetilde{X}_{s}$. More precisely, the elements of $\widetilde{X}_{s}^{(d)}$ can be written as

$$
\widetilde{x}_{i j}^{(d)}=\delta_{i j} \tilde{x}_{i j},
$$

where $\delta_{i j}$ is Kronecker's delta and $\widetilde{x}_{i j}$ are the elements of $\widetilde{X}_{s}$. With this new choice of the matrix $L$, the LMI optimization problem $\mathscr{P}_{o}$ with the structure constraints set in (50) can be properly solved and it produces the velocity-feedback gain matrix:

$$
\begin{aligned}
\widetilde{K}_{d}=10^{6} & \\
& \times\left[\begin{array}{ccccc}
-5.8490 & 0 & 0 & 0 & 0 \\
0 & -5.5039 & 0 & 0 & 0 \\
0 & 0 & -4.9631 & 0 & 0 \\
0 & 0 & 0 & -4.7182 & 0 \\
0 & 0 & 0 & 0 & -4.6619
\end{array}\right] .
\end{aligned}
$$

In this case, the associated $\gamma$-value satisfies

$$
\gamma_{\widetilde{G}_{d}} \leq 0.0454
$$

where

$$
\widetilde{G}_{d}=\widetilde{K}_{d} C_{v}
$$

is the state gain matrix associated with the decentralized velocity-feedback controller.

Remark 2. It should be highlighted that the value 0.0454 in (54) is just an upper bound [21]. The actual value of $\gamma_{\widetilde{\mathrm{G}}_{d}}$ can be computed by considering the LMIs,

$$
\begin{gathered}
\left(A+B \widetilde{G}_{d}\right) X+X\left(A+B \widetilde{G}_{d}\right)^{T}+E E^{T}<0, \\
\left(C_{z}+D_{z} \widetilde{G}_{d}\right) X\left(C_{z}+D_{z} \widetilde{G}_{d}\right)^{T}-\eta I<0,
\end{gathered}
$$

and solving the auxiliary LMI optimization problem:

$$
\mathscr{P}_{a}:\left\{\begin{array}{l}
\text { minimize } \eta \\
\text { subject to } X>0, \eta>0, \\
\text { and the LMIs in }(56) .
\end{array}\right.
$$

If $\mathscr{P}_{a}$ admits the optimal solution $\widetilde{\eta}_{a}$, then we have

$$
\gamma_{\widetilde{G}_{d}}=\widetilde{\eta}_{a}^{1 / 2} .
$$

In our case, we obtain the $\gamma$-value:

$$
\gamma_{\widetilde{\mathrm{G}}_{d}}=0.0407,
$$

which is just a $3 \%$ larger than the optimal $\gamma$-value in (29) attained by the ideal state-feedback controller.

Remark 3. The control forces exerted by the fully decentralized velocity-feedback controller in (48) take the form

$$
u_{i}(t)=k_{i i} v_{i}(t), \quad i=1, \ldots, 5 .
$$

If the actuation devices $a_{i}, i=1, \ldots, 5$, in Figure 1 are assumed to be linear dampers with respective damping constants $b_{i}>$ $0, i=1, \ldots, 5$, then the actuation forces produced by the passive-damping system are

$$
u_{i}(t)=-b_{i} v_{i}(t), \quad i=1, \ldots, 5 .
$$

When all the coefficients $k_{i i}$ are negative, the control forces in (60) can be exerted by a system of interstory linear dampers with damping constants:

$$
b_{i}=-k_{i i}, \quad i=1, \ldots, 5 .
$$

Consequently, the decentralized velocity-feedback energyto-peak controller defined by the gain matrix $\widetilde{K}_{d}$ can be implemented by a set of linear passive dampers, with no sensors, no communication system, and null power consumption [19].

To demonstrate the good behavior of the passivedamping system defined by the gain matrix $\widetilde{K}_{d}$, the vibrational response of the five-story building has been numerically simulated for this passive control configuration, taking again the full scale North-South 1940 El Centro seismic record as ground acceleration input. The maximum absolute interstory drifts and the maximum absolute control efforts corresponding to the passive-damping system are displayed in Figures 5 and 6, respectively, using a red line with asterisks. In both figures, the values corresponding to the ideal statefeedback controller designed in Section 3 are displayed by a blue line with circles. From the graphics in Figure 5, it can be clearly appreciated that the levels of reduction in the interstory drifts peak-values attained by the passive-damping system are similar to those obtained by the ideal active statefeedback controller. Looking at the graphics in Figure 6, it can also be appreciated that similar, or even lower, control effort peak-values are produced by the proposed passive-damping system.

\section{Conclusions and Future Directions}

In this paper, we have presented a new design strategy that makes it possible to synthesize fully decentralized velocityfeedback energy-to-peak controllers by solving two successive LMI optimization problems. By taking advantages of the 


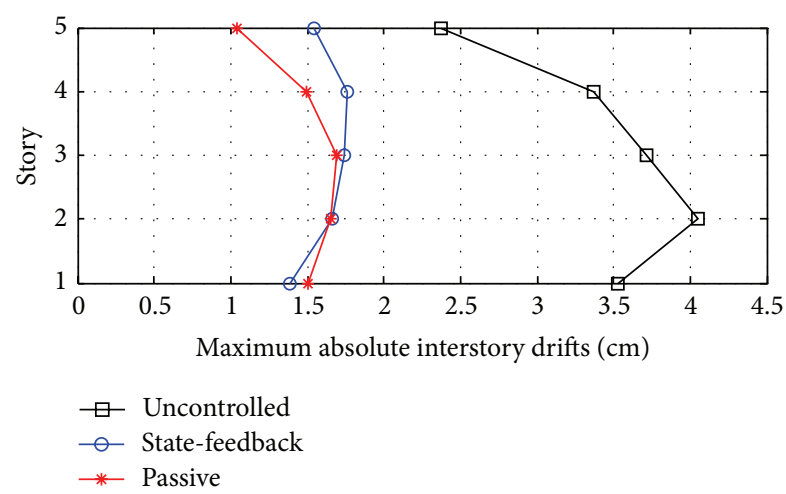

FIGURE 5: Maximum absolute interstory drifts corresponding to the uncontrolled building (black line with rectangles), the ideal statefeedback energy-to-peak controller defined by the gain matrix $\widetilde{G}_{s}$ (blue line with circles), and the passive-damping system defined by the gain matrix $\widetilde{K}_{d}$ (red line with asterisks). The full scale NorthSouth El Centro 1940 seismic record has been used as ground acceleration disturbance.

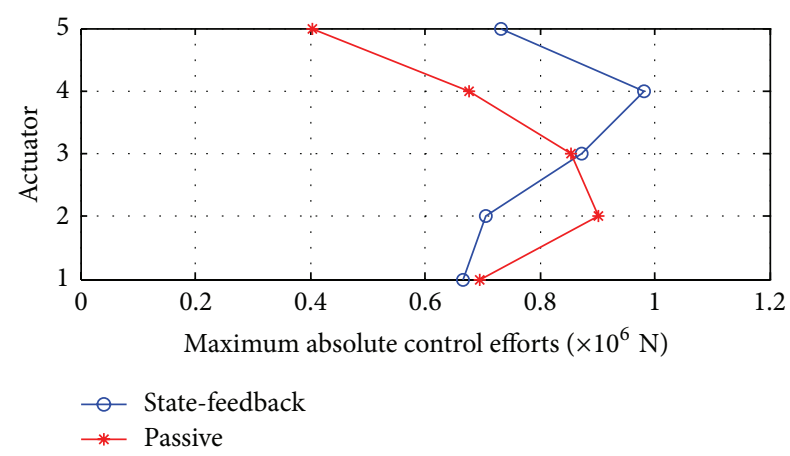

FIGURE 6: Maximum absolute control efforts corresponding to the ideal state-feedback energy-to-peak controller defined by the gain matrix $\widetilde{G}_{s}$ (blue line with circles) and the passive-damping system defined by the gain matrix $\widetilde{K}_{d}$ (red line with asterisks). The full scale North-South El Centro 1940 seismic record has been used as ground acceleration disturbance.

link between fully decentralized velocity-feedback controllers and passive linear dampers, advanced active feedback control strategies can be used to design complex passive-damping systems for vibration control of large structures. The passivedamping systems so obtained combine the simplicity and robustness of passive control systems with the effectiveness of active feedback control. To demonstrate the main features of the new design methodology, a passive-damping system for the seismic protection of a five-story building has been designed with excellent results. It has to be pointed out, however, that no satisfactory solutions are produced by the proposed design strategy in the case of fully decentralized velocity-feedback controllers under the $H_{\infty}$ approach and, consequently, further research attention should be paid to this relevant problem. As other important lines of future work, we can also include limited frequency domain [32], multistructure systems $[33,34]$, robust strategies, and adaptive strategies $[35,36]$.

\section{Conflict of Interests}

The authors declare that there is no conflict of interests regarding the publication of this paper.

\section{Acknowledgments}

This work was partially supported by the Spanish Ministry of Economy and Competitiveness through the Grant DPI201232375/FEDER and by the Norwegian Center of Offshore Wind Energy (NORCOWE) under Grant 193821/S60 from the Research Council of Norway (RCN). NORCOWE is a consortium with partners from industry and science, hosted by Christian Michelsen Research. The work was also partially supported by a Grant from Iceland, Liechtenstein, and Norway through the EEA Financial Mechanism, operated by the Universidad Complutense de Madrid.

\section{References}

[1] B. F. Spencer Jr. and S. Nagarajaiah, "State of the art of structural control," Journal of Structural Engineering, vol. 129, no. 7, pp. 845-856, 2003.

[2] H. Li and L. Huo, "Advances in structural control in civil engineering in China," Mathematical Problems in Engineering, vol. 2010, Article ID 936081, 23 pages, 2010.

[3] B. Basu, O. S. Bursi, F. Casciati et al., "A European Association for the Control of Structures joint perspective. Recent studies in civil structural control accross Europe," Structural Control and Health Monitoring, 2014.

[4] Y. Wang, J. P. Lynch, and K. H. Law, "Decentralized $\mathscr{H}_{\infty}$ controller design for large-scale civil structures," Earthquake Engineering and Structural Dynamics, vol. 38, no. 3, pp. 377-401, 2009.

[5] F. Palacios-Quiñonero, J. M. Rossell, and H. R. Karimi, "Semidecentralized strategies in structural vibration control," Modeling, Identification and Control, vol. 32, no. 2, pp. 57-77, 2011.

[6] Y. Lei, D. T. Wu, and Y. Lin, "A decentralized control algorithm for large-scale building structures," Computer-Aided Civil and Infrastructure Engineering, vol. 27, no. 1, pp. 2-13, 2012.

[7] F. Palacios-Quiñonero, J. Rubió-Massegú, J. M. Rossell, and H. R. Karimi, "Discrete-time multioverlapping controller design for structural vibration control of tall buildings under seismic excitation," Mathematical Problems in Engineering, vol. 2012, Article ID 636878, 20 pages, 2012.

[8] H. R. Karimi, F. Palacios-Quiñonero, J. M. Rossell, and J. RubióMassegú, "Sequential design of multioverlapping controllers for structural vibration control of tall buildings under seismic excitation," Proceedings of the Institution of Mechanical Engineers I: Journal of Systems and Control Engineering, vol. 227, no. 2, pp. 176-183, 2013.

[9] M. Sonmez, E. Aydin, and T. Karabork, "Using an artificial bee colony algorithm for the optimal placement of viscous dampers in planar building frames," Structural and Multidisciplinary Optimization, vol. 48, no. 2, pp. 395-409, 2013.

[10] N. Gluck, A. M. Reinhorn, J. Gluck, and R. Levy, "Design of supplemental dampers for control of structures," Journal of Structural Engineering, vol. 122, no. 12, pp. 1394-1399, 1996.

[11] A. K. Agrawal and J. N. Yang, "Design of passive energy dissipation systems based on LQR control methods," Journal of 
Intelligent Material Systems and Structures, vol. 10, no. 12, pp. 933-944, 1999.

[12] J. N. Yang, S. Lin, J.-.H. Kim, and A. K. Agrawal, "Optimal design of passive energy dissipation systems based on $H_{\infty}$ and $\mathrm{H}_{2}$ performances," Earthquake Engineering and Structural Dynamics, vol. 31, no. 4, pp. 921-936, 2002.

[13] D. D. Moerder and A. J. Calise, "Convergence of a numerical algorithm for calculating optimal output feedback gains," IEEE Transactions on Automatic Control, vol. 30, no. 9, pp. 900-903, 1985.

[14] V. L. Syrmos, C. T. Abdallah, P. Dorato, and K. Grigoriadis, "Static output feedback-a survey," Automatica, vol. 33, no. 2, pp. 125-137, 1997.

[15] Y. Cao, L. James, and Y. Sun, "Static output feedback stabilization: an ILMI approach," Automatica, vol. 34, no. 12, pp. 1641$1645,1998$.

[16] Z. Shu and J. Lam, "An augmented system approach to static output-feedback stabilization with $H_{\infty}$ performance for continuous-time plants," International Journal of Robust and Nonlinear Control, vol. 19, no. 7, pp. 768-785, 2009.

[17] F. Palacios-Quiñonero, J. Rubió-Massegú, J. M. Rossell, and H. R. Karimi, "Feasibility issues in static output-feedback controller design with application to structural vibration control," Journal of the Franklin Institute, vol. 351, no. 1, pp. 139-155, 2014.

[18] J. Rubió-Massegú, J. M. Rossell, H. R. Karimi, and F. PalaciosQuiñonero, "Static output-feedback control under information structure constraints," Automatica, vol. 49, no. 1, pp. 313-316, 2013.

[19] F. Palacios-Quiñonero, J. Rubió-Massegú, J. M. Rossell, and H. R. Karimi, "Optimal passive-damping design using a decentralized velocity-feedback $H_{\infty}$ approach," Modeling, Identification and Control, vol. 33, no. 3, pp. 87-97, 2012.

[20] J. Rubió-Massegú, F. Palacios-Quiñonero, and J. M. Rossell, "Decentralized static output-feedback $H_{\infty}$ controller design for buildings under seismic excitation," Earthquake Engineering \& Structural Dynamics, vol. 41, no. 7, pp. 1199-1205, 2012.

[21] F. Palacios-Quiñonero, J. Rubió-Massegú, J. M. Rossell, and H. R. Karimi, "Vibration control for adjacent structures using local state information," Mechatronics, vol. 24, no. 4, pp. 336-344, 2014.

[22] G. J. Balas, R. Y. Chiang, A. K. Packard, and M. G. Safonov, MATLAB Robust Control Toolbox 3. Users Guide, Version 4.2, The MathWorks, Inc., Natick, Mass, USA, 2012.

[23] H. Du and J. Lam, "Energy-to-peak performance controller design for building via static output feedback under consideration of actuator saturation," Computers and Structures, vol. 84, no. 31-32, pp. 2277-2290, 2006.

[24] W. Zhang, Y. Chen, and H. Gao, "Energy-to-peak control for seismic-excited buildings with actuator faults and parameter uncertainties," Journal of Sound and Vibration, vol. 330, no. 4, pp. 581-602, 2011.

[25] H. Zhang, Y. Shi, A. S. Mehr, and H. Huang, "Robust FIR equalization for time-varying communication channels with intermittent observations via an LMI approach," Signal Processing, vol. 91, no. 7, pp. 1651-1658, 2011.

[26] H. Zhang, X. Zhang, and J. Wang, "Robust gain-sheduling energy-to-peak control of vehicle lateral dynamics stabilisation,” Vehicle System Dynamics, vol. 52, no. 3, pp. 309-340, 2014.

[27] H. Zhang, Y. Shi, and J. Wang, "On energy-to-peak filtering for nonuniformly sampled nonlinear systems: a markovian jump system approach," IEEE Transactions on Fuzzy Systems, vol. 22, no. 1, pp. 212-222, 2014.
[28] Q. Chenggang, C. Xibin, H. R. Karimi, Z. Zhang, and Z. Zexu, "Finite-time distributed energy-to-peak control for uncertain multiagent systems," Abstract and Applied Analysis, vol. 2014, Article ID 260201, 9 pages, 2014.

[29] N. Kurata, T. Kobori, M. Takahashi, N. Niwa, and H. Midorikawa, "Actual seismic response controlled building with semi-active damper system," Earthquake Engineering and Structural Dynamics, vol. 28, no. 11, pp. 1427-1447, 1999.

[30] A. K. Chopra, Dynamics of Structures. Theory and Applications to Earthquake Engineering, Prentice Hall, Upper Saddle River, NJ, USA, 3rd edition, 2007.

[31] H. Kurino, Y. Matsunaga, T. Yamada, and J. Tagami, "High performance passive hydraulic damper with semi-active characteristics," in Proceedings of the 13th World Conference on Earthquake Engineering, Paper no. 33, Vancouver, Canada, August 2004.

[32] Y. Chen, W. Zhang, and H. Gao, "Finite frequency $H_{\infty}$ control for building under earthquake excitation," Mechatronics, vol. 20, no. 1, pp. 128-142, 2010.

[33] F. Palacios-Quiñonero, J. M. Rossell, J. Rubió-Massegú, and H. R. Karimi, "Structural vibration control for a class of connected multistructure mechanical systems," Mathematical Problems in Engineering, vol. 2012, Article ID 942910, 23 pages, 2012.

[34] F. Palacios-Quiñonero, J. Rubió-Massegú, J. M. Rossell, and H. R. Karimi, "Semiactive-passive structural vibration control strategy for adjacent structures under seismic excitation," Journal of the Franklin Institute, vol. 349, no. 10, pp. 3003-3026, 2012.

[35] H. Du, J. Lam, and K. Y. Sze, "Non-fragile $H_{\infty}$ vibration control for uncertain structural systems," Journal of Sound and Vibration, vol. 273, no. 4-5, pp. 1031-1045, 2004.

[36] H. Du, N. Zhang, and F. Naghdy, "Actuator saturation control of uncertain structures with input time delay," Journal of Sound and Vibration, vol. 330, no. 18-19, pp. 4399-4412, 2011. 


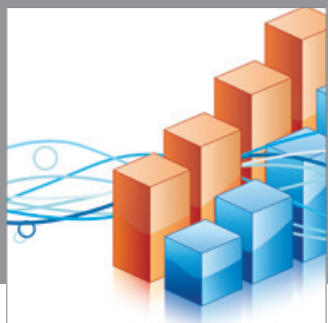

Advances in

Operations Research

mansans

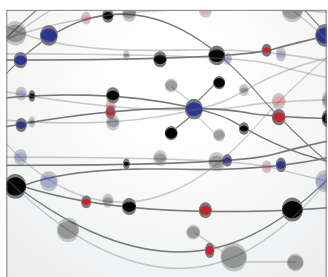

The Scientific World Journal
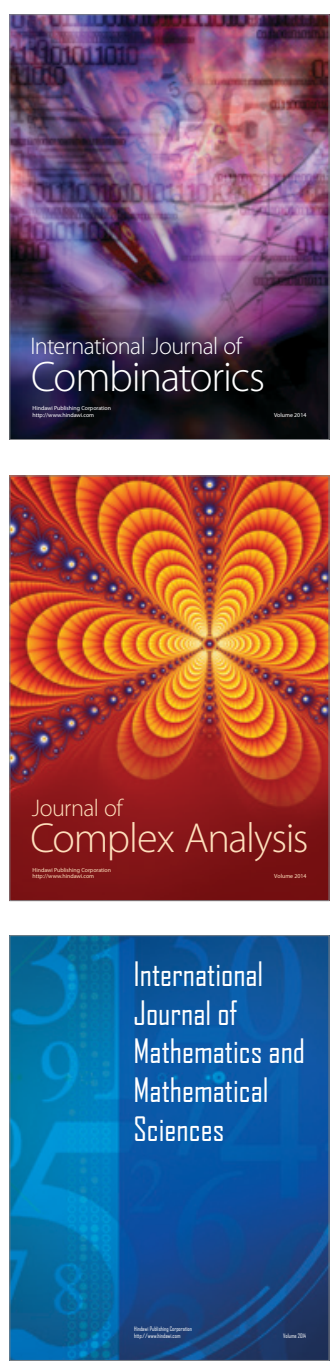
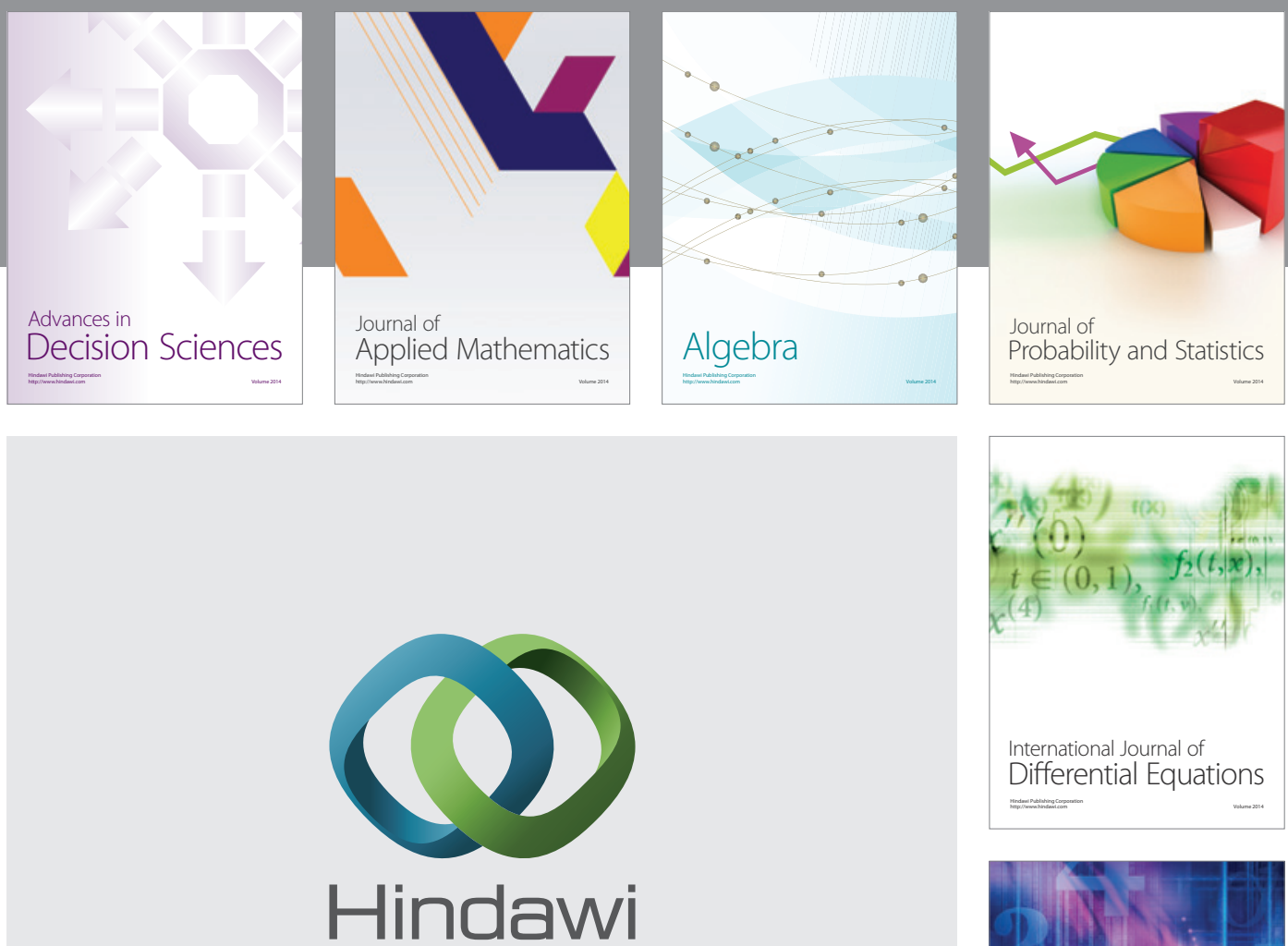

Submit your manuscripts at http://www.hindawi.com
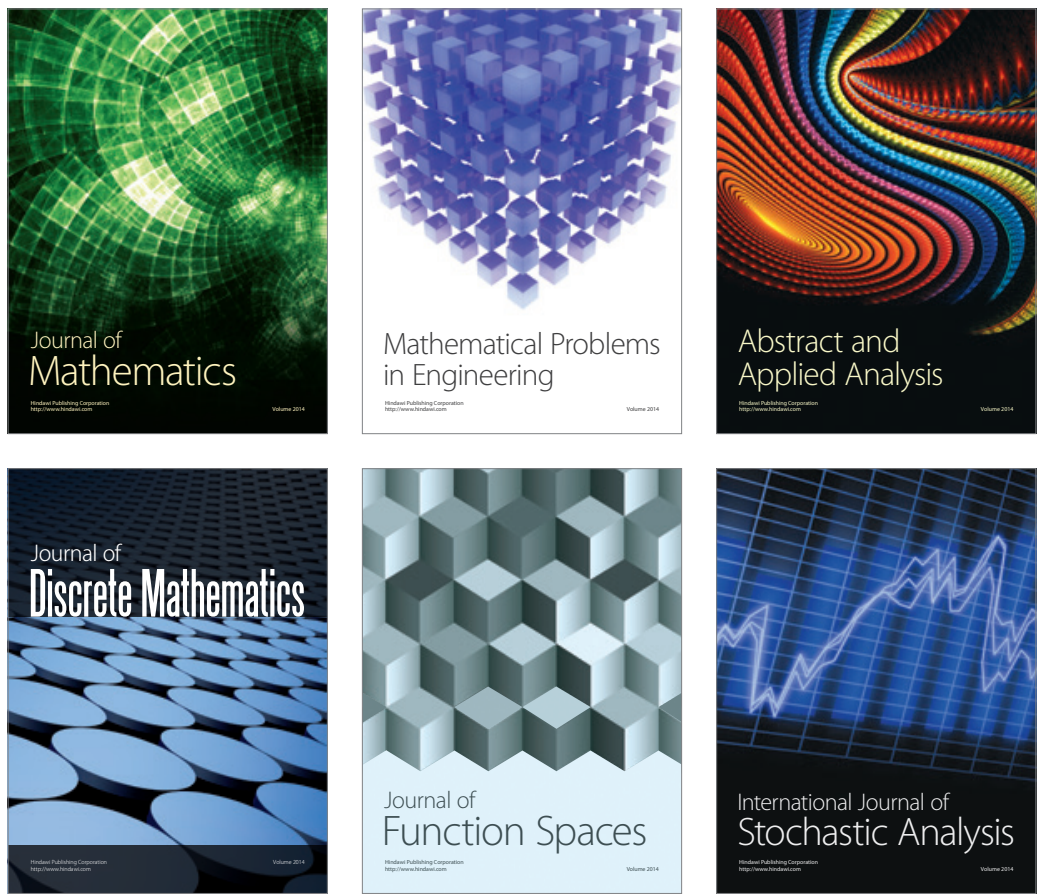

Journal of

Function Spaces

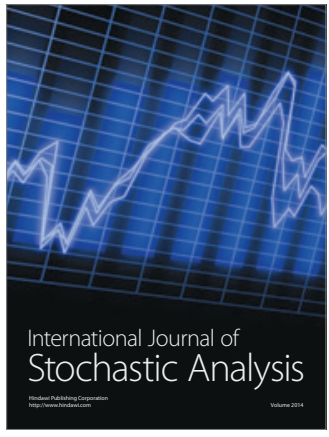

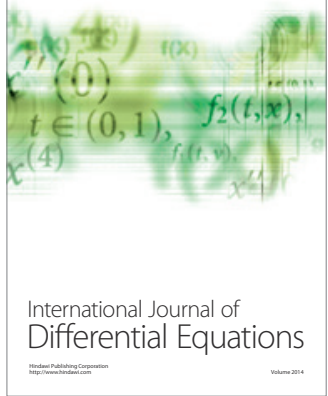
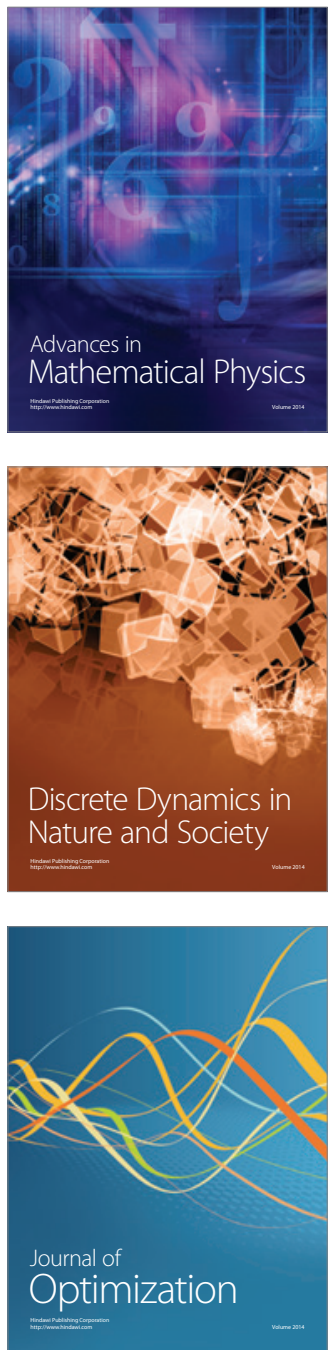